\title{
Ion-Induced Electron Emission from MgO by Exciton Decay into Vacuum
}

\author{
M. Ishimoto ${ }^{1,2}$, R.A. Baragiola ${ }^{2}$, P.Riccardi $^{2,3}$ and T. Shinoda ${ }^{1}$ \\ ${ }^{1}$ Fujitsu Laboratories Ltd., Akashi, Hyogo, 674-8555, Japan \\ ${ }^{2}$ University of Virginia, Laboratory for Atomic and Surface Physics \\ Charlottesville, VA 22904, U. S. A. \\ ${ }^{3}$ Laboratorio IIS, Dipartimento di Fisica, Universitá della Calabria, and INFM Unitá di Cosenza, 87036 \\ Arcavacata di Rende, Cosenza, Italy
}

\begin{abstract}
We report observations of electron emission from $\mathrm{MgO}$ surfaces induced by impact of $100 \mathrm{eV}-4 \mathrm{keV}$ $\mathrm{He}+, \mathrm{Na}+, \mathrm{Ne}+, \mathrm{Ar}+$ ions. The energy distribution of emitted electrons is nearly independent of ion type and energy, showing that it is caused by the decay of an intrinsic electronic state of the solid excited by the ions. The similar yields and energy distributions for incident $\mathrm{Na}+$ and $\mathrm{Ne}+$ ions rule out the potential mechanism of electron emission. The results are consistent with a novel model in which excitation occurs when electrons centered at the oxygen anions are promoted during a collision with the projectile (kinetic mechanism), and transferred to a surface exciton that can autoionize since it lies above the vacuum level, as determined by combining measurements of electron energy loss and photoelectric threshold.
\end{abstract}

Keywords: electron emission, magnesium oxide, ionsurface collisions, negative electron affinity, surface band gap

\section{Introduction}

$\mathrm{MgO}$ is widely used as the protective layer in Plasma Display Panel (PDP) because it has good characteristics of secondary electron emission. However it is not understood clearly why MgO has such a good ability of electron emission.

Electron emission is a fundamental consequence of the interaction of slow ions with solid surfaces. It is understood within the general frameworks of the kinetic and potential emission mechanisms, ${ }^{1}$ in which the energy required to free electrons is provided, respectively, by the motion of the incoming ion or by its potential energy. The vast majority of the studies on ion-induced electron emission have been performed using metal targets. Insulator surfaces, of have high relevance in both basic research and technology, have received considerable less attention ${ }^{2,3}$ due to experimental difficulties. The mechanism of potential emission, Auger neutralization, an electron from the solid neutralizes the projectile and the excess energy is given to another electron of the solid. For ionic insulators, of large ionization energy $I$ (sum of bandgap $E_{\mathrm{g}}$ and electron affinity $A$ ), Auger neutralization cannot occur for incident ions like $\mathrm{Na}^{+}$that have a neutralization energy $E_{\mathrm{n}}<2 I+\varepsilon$, where $\varepsilon$ is the interaction energy between the final two holes in the valence band. Surprisingly, the yield of electrons from these ionic solids induced by slow ions has been found to be larger than for metals, ${ }^{2-5}$ even though in metals less energy is required to remove an electron (the work function). In addition, electron emission from ionic insulators has been observed at impact energies below $100 \mathrm{eV}^{4-8}$ in contrast to electron emission from metals, which is strongly reduced for impact energies below $0.5-2 \mathrm{keV}{ }^{1}$ The reasons for this dissimilar behavior have not been elucidated.

\section{Experiments and Results}

To examine the question of enhanced electron emission in the interactions of slow ions with insulators, we measured the energy distributions of electrons emitted from an $\mathrm{MgO}$ surface under the impact of slow noble gas and sodium ions. The study of magnesium oxide, a simple ionic crystal, is justified not only from the point of view of fundamental physics — the basic interactions leading to electron emission from $\mathrm{MgO}$ are not well known ${ }^{9,10}$ - but also because of advantageous properties of this material for use in plasma display panels: a large ion-induced electron yield (and therefore a low discharge voltage ${ }^{11}$ ) and high stability under ion bombardment. ${ }^{12}$

The experiments were performed in an ultrahigh vacuum $\left(\sim 10^{-10}\right.$ Torr) surface science system used in previous electron emission studies. ${ }^{13}$ Electrons ejected from the MgO samples were energy analyzed with double-pass cylindrical mirror spectrometer operated inside a magnetic shield at constant pass energy of $50 \mathrm{eV}$ and a resolution of $0.2 \mathrm{eV}$. The surface of the samples was normal to the ion beam and at $78^{\circ}$ with respect to the spectrometer axis. Sodium ions were produced in a thermal ionization source, and noble gas ions in an electron impact source, operated at $58 \mathrm{eV}$ electron energy.

The MgO films, about $100 \mathrm{~nm}$ thick, were prepared by electron beam deposition on a highly doped Si substrate. They are polycrystalline with the grains oriented such that they present an oxygen-terminated (111) surface. The samples were sputter cleaned with $1 \mathrm{keV} \mathrm{Ar}^{+}$and the cleaning was monitored by Auger Electron Spectroscopy (AES) and Electron Energy Loss Spectroscopy (EELS). Weak features in the EELS spectra due to band-gap states disappeared after cleaning. ${ }^{12}$ AES and EELS spectra for the sputter cleaned MgO surfaces were in excellent agreement with published spectra ${ }^{14-16}$ of clean $\mathrm{MgO}$; the absence of significant band-gap states showed that sputter cleaning produced negligible damage. Normal electron emission measurements are not possible with an insulator like MgO since the surface electrostatic potential becomes positive as ions deposit their charge on the surface and electrons are emitted into vacuum. The positive surface potential produces a time-dependent distortion in the energy spectra. To avoid or minimize this problem we used low ion fluxes $\left(<100 \mathrm{nA} / \mathrm{cm}^{2}\right)$ and neutralized the surface charge immediately before the acquisition of each spectrum by flooding the surface with low energy electrons from a nearby filament (an electron flood gun used routinely for 


\section{Ishimoto}

charge compensation in photoelectron spectroscopy of insulators). The neutralization procedure reduced the surface potential below $0.2 \mathrm{~V}$. This is demonstrated by the quick rise in the energy distributions of electrons very close to the voltage at which the sample is biased $(-4.9 \mathrm{~V})$, and by the constancy of the AES energies measured after exposing the sample to the electron flood gun. Repeated energy spectral scans showed that at the ion current densities used neither surface charging nor damage had significant effects over the $\sim 20$ second spectrum acquisition time.

Our results show that, unexpectedly, the energy distribution of electrons emitted by slow $\mathrm{He}^{+}, \mathrm{Na}^{+}, \mathrm{Ne}^{+}$, $\mathrm{Ar}^{+}$, and $\mathrm{Xe}^{+}$are very similar. This unexpected behavior is explained with a model in which oxygen- $2 p$ electrons are promoted in a close collision between the projectile and the anion, populating an exciton level that decays into vacuum due to the negative electron affinity of MgO. Similar results were obtained using $30 \mathrm{eV}$ electrons in the ion source, indicating negligible contamination of the ion beam with doubly charged ions.

Fig. 1 shows $N(E)$, the energy spectra of electrons emitted from $\mathrm{MgO}$ by $200 \mathrm{eV} \mathrm{He}{ }^{+}, \mathrm{Ne}^{+}$, and $\mathrm{Ar}^{+}$ions, and $500 \mathrm{eV}$ $\mathrm{Na}^{+}$ions.. The total electron yields $\gamma$ shown in fig.2, were obtained from the currents measured on the sample under positive and negative bias, with an uncertainty of $\sim 30 \%$. The fact that the yields for $\mathrm{He}^{+}$are larger than for $\mathrm{Ar}^{+}$ (which sputters much more effectively) clearly indicates that emission of negative ions has a negligible contribution to the yields (the $\mathrm{O}^{-}$yields are below 0.1 for $300 \mathrm{eV} \mathrm{Na}^{+}$ impacting other oxides). ${ }^{17}$ The increase of the total electron yield above unity for high energy $\mathrm{He}^{+}$signals the contribution of multiple excitation collisions during penetration.

Fig. 1 shows that the shape of the energy distributions is nearly the same for the different ions, in remarkable contrast with the large variations observed for collisions with metal samples. ${ }^{1,18}$ This finding allows us to exclude emission mechanisms that involve the electron levels of the projectile ions, such as Auger neutralization (AN), ${ }^{1}$ that have been often invoked as a source of electrons in slow ion interactions with $\mathrm{MgO} .{ }^{19}$ In Auger neutralization, the maximum electron energy is $E_{\mathrm{n}}-2 I,{ }^{1,18}$ where the ionization energy of $\mathrm{MgO}$ is $I=5.5 \mathrm{eV}$ at the surface (Fig. 3), and $E_{\mathrm{n}}$, the ionization potential of the parent atoms, varies from $24.58 \mathrm{eV}$ to $5.14 \mathrm{eV}$ going from He to $\mathrm{Na}$. In all cases, the ionization potential increases when the projectile is in the potential of a $\mathrm{Mg}^{++}$ion but the large $(19.44 \mathrm{eV})$ difference in available potential energy would subside making the AN spectra strongly dependent on the projectile type. The independence of the spectra on the type of projectile also rules out other mechanisms, like double electron capture to a negative ion followed by electron detachment in a third collision, ${ }^{20}$ the decay of a transient autoionization complex of the incident ion and two adjacent target atoms. ${ }^{21}$

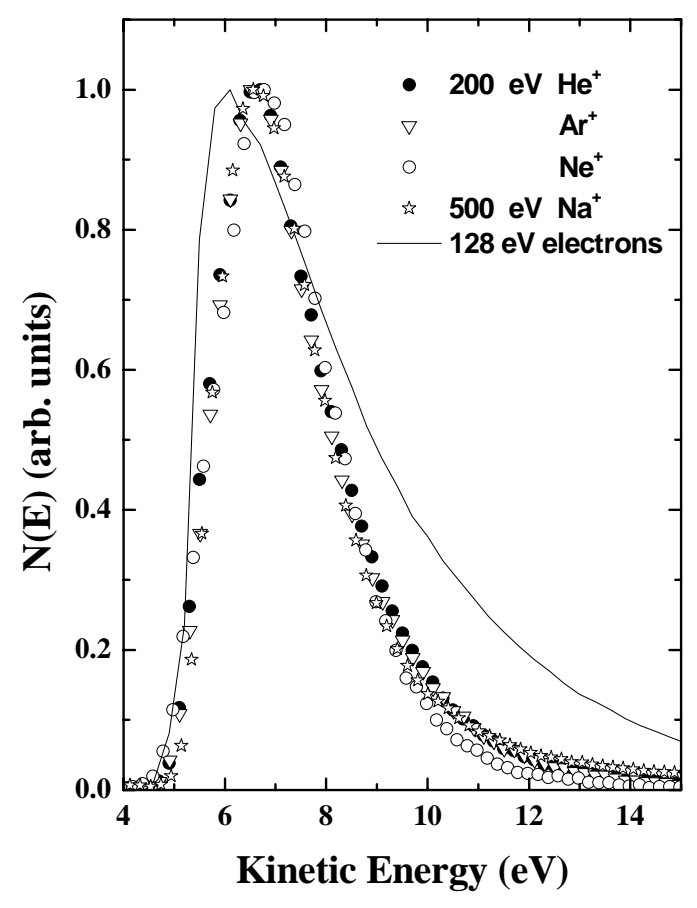

Figure 1. Normalized energy distribution of electrons ejected from a MgO surface under impact of $200 \mathrm{eV}$ noble gas ions and $128 \mathrm{eV}$ electrons. The sample was biased at $-4.9 \mathrm{eV}$.

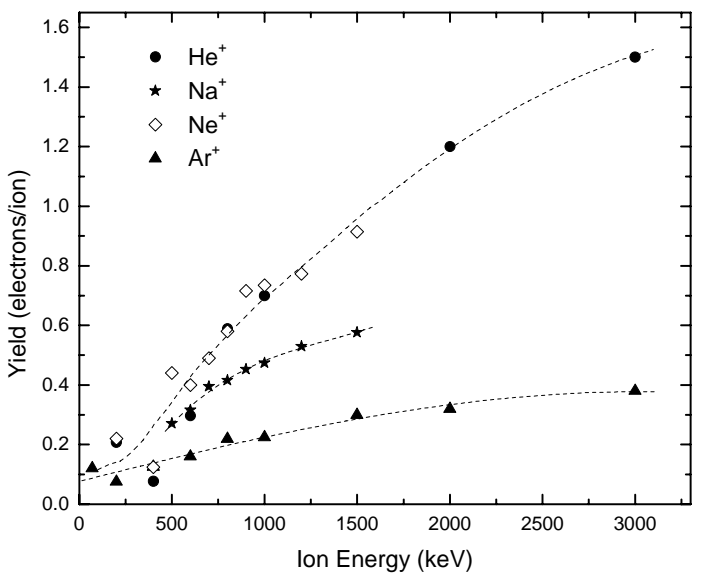

Figure 2. Energy dependence of the total electron yields from a $\mathrm{MgO}(111)$ surface at normal incidence. Experimental uncertainties are 20\%. 


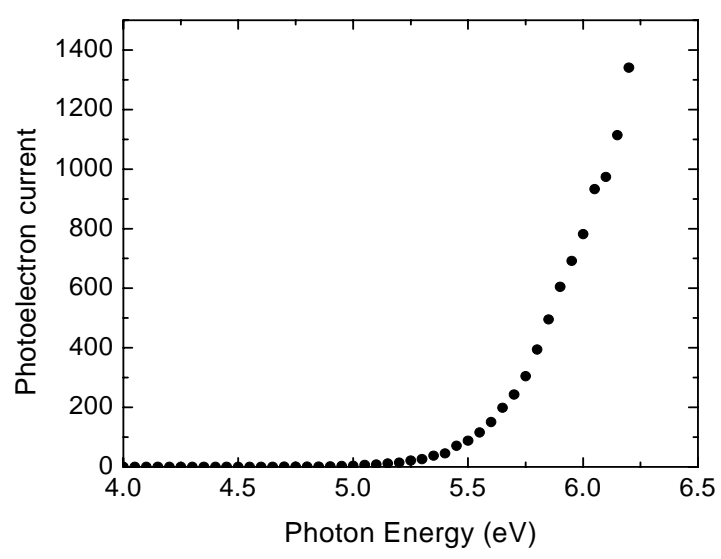

Figure 3. Photoelectric threshold of a $\mathrm{MgO}(111)$ surface.

We note in fig. 1 that the similar ion-excited electron spectra do not result from a peculiar density of final electronic states, since the spectra are quite different to that excited by incident electrons (Fig. 1). This suggests that electron emission results from the preferential excitation by ions of a state intrinsic to the solid, i.e., not involving the electronic configuration or energy of the projectile. An intrinsic mechanism was suggested recently by Matulevich et al. $^{22}$ for $50 \mathrm{eV}$ noble gas ions incident on much thinner (1-5 nm) MgO films grown on oxidized Mo. In this mechanism, the incident ions undergo Auger neutralization producing valence band holes that move to the substrate and eject electrons from it by Auger neutralization. In these experiments, the surface charges by $\sim 2 \mathrm{eV}$, as judged by their energy diagram. The resulting electric field may indeed drive holes to the substrate and assist in electron ejection. ${ }^{23}$ However, the electron yields, much higher than those reported here, are inconsistent with Auger neutralization from oxidized Mo, and the authors did not find the independence of the electron spectra on type of projectile. In addition, their model cannot account for very similar electron yields produced by $\mathrm{He}^{+}, \mathrm{Na}^{+}$and $\mathrm{Ne}^{+}$ions.

\section{Discussion}

We propose excitation occurs by the well-known electron promotion effect in atomic collisions, which occurs due to electron-electron interaction in the interpenetration of the valence shells of the projectile and a target atom (oxygen anion) during a close collision, forming a transient quasi molecule. Electron promotion is allowed by the kinetic energy of the projectile and hence the electron emission process it affords is of the kinetic type. This atomistic description of ion-surface interactions is adequate because the valence charge is localized at the anion sites in $\mathrm{MgO}$. The binary interaction promotes oxygen- $2 p$ electrons along quasi-molecular orbitals (MOs), ${ }^{24}$ above a threshold projectile energy which has been determined to be $\sim 50 \mathrm{eV}$ for the analogous case of $\mathrm{Na}^{+}$exciting oxidized $\mathrm{Al}^{17}$ Electrons in these promoted MOs: 3d $\sigma$ for He-O, $4 \mathrm{f} \sigma$ for Na-O, Ne-O [Ref. 25] and Ar-O can be transferred to excited states when the promoted MO crosses unfilled
MOs that correlate to excitons and conduction band states. The population of the final excited levels will decrease with excitation energy $\Delta E$; and therefore will most likely lead to excitonic states. Promotion crossing the continuum may contribute $[7,8]$, together with $\mathrm{MO}$ autoionization, to the high-energy tail of the electron spectrum.

We propose that the excitons populated by electron promotion contribute to electron emission because they decay into vacuum. Excitons are normally considered to be bound states of solids but, in $\mathrm{MgO}$ (and in $\mathrm{LiF}$ ), the negative electron affinity of the surface causes the exciton to be above the vacuum level. ${ }^{26}$ The band gap of MgO (7.8 $\mathrm{eV}$ in the bulk) ${ }^{12}$ drops at the surface due to the decrease in the Madelung potential, by more than $1 \mathrm{eV}$, with variations among different crystal faces. ${ }^{27,28}$ EELS measurements show a minimum surface excitation energy of $5.5 \mathrm{eV},{ }^{26}$ and a decrease in the exciton energy from a bulk value of 7.7 $\mathrm{eV}$ to $\sim 6.2 \mathrm{eV}$, for $\mathrm{MgO}(100){ }^{12}$ The surface exciton exists in a region of changing surface potential extending from outside the solid to the first atomic layers. ${ }^{26}$ To establish the location of the vacuum level, we determined the ionization energy of our samples (energy difference between the top of the valence band and the vacuum level) by measuring the photoelectron threshold (Fig. 3). The value of $\sim 5.3 \mathrm{eV}$ is nearly the same as the minimum excitation energy measured in EELS, showing that the surface exciton is above the vacuum level, which can explain its unusually large width. With this information we construct the approximate energy level diagram for the MgO surface, shown in Fig. 4, that includes the decrease in conduction band energies and increase in valence band energies at the surface that have resulted from detailed calculations. $^{29}$

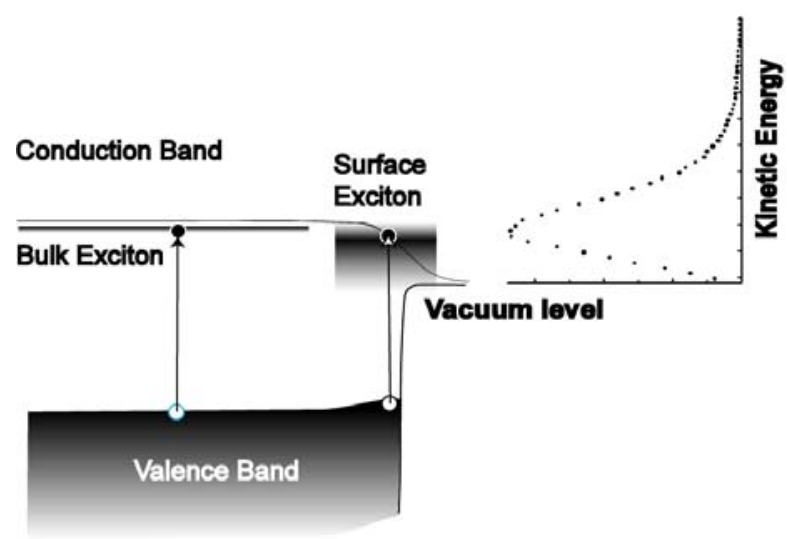

Exciton autoionization on negative electron affinity $\mathrm{MgO}$ surface

Figure 4. Schematic energy levels for a $\mathrm{MgO}(111)$ surface, showing how the decay of an exciton, allowed by the negative electron affinity, gives rise to the observed energy distribution of emitted electrons. The block labeled 'surface exciton' represents the range of surface energy losses measured in EELS, and its extension from outside the solid to the first surface layer.26 


\section{Ishimoto}

The negative electron affinity of the surface (vacuum level below the bulk conduction band minimum) allows excitons to couple to the continuum of states outside the solid, which explains the considerable width of surface excitons. $^{26}$ The decay of the excitons into vacuum (or exciton break-up) ${ }^{30,31}$ then produces electrons with an energy distribution peaked at the observed low energies, due to the small (positive) difference in energy between the peak exciton energy and the vacuum level. In conclusion, the energy distribution of electrons emitted from $\mathrm{MgO}$ surfaces under the impact of slow singly charged noble gas ions shows a remarkable independence of the type of incident ion. This result shows that electron emission results from the decay of an intrinsic excitation in the solid. We propose that excitons produced by electron promotion during binary projectile-oxygen collisions decay into vacuum due to the negative electron affinity of the surface.

As we described here, MgO has very unique property of electron emission because of the negative electron affinity. This electron emission mechanism will also help to understand of the exoelectron emission from MgO.

\section{References}

1. R.A. Baragiola in Low Energy Ion-Surface Interactions, Edited by J.W. Rabalais, Wiley, New York, 1994-chap. 4

2. J. Schou, in Ionization of Solids by Heavy Particles, edited by R. A. Baragiola (Plenum New York, 1993), p. 351.

3. H. Winter, Prog. Surf. Sci. 63, 177 (2000).

4. U.A. Arifov, R.R. Rakhimov, S. Gaipov, Radio Engineering and Electronic Physics 15, 6 (1970) 1059.

5. Y. Ushio, T. Banno, N. Matuda, Y Saito, S. Bada, A. Kinbara, Thin Solid Films 167 (1988) 299.

6. M. Vana, F. Aumayr, P. Varga, HP. Winter Europhys. Lett. 29, 55 (1995).

7. P. Stracke et al. Nucl. Inst. and Meth. Phys. Res. B 125, 67 (1997).

8. P.A. Zaijlmans van Emmichoven et al., Phys. Rev. B 59, 10950 (1999).

9. S. Ustaze, R. Verucchi, S. Lacombe, L. Guillemot, V.A. Esaulov Phys. Rev. Lett 79, 3526 (1997).

10. D. Ochs et al. Surf. Sci. 365, 557 (1996).

11. H.S. Uhm, E.H. Choi and G.S. Cho, Appl. Phys. Lett. 78, 592 (2001).
12. V.E. Henrich, P.A. Cox, The Surface Science of metal Oxides (Cambridge University Press, Cambridge, England, 1994).

13. R.A. Baragiola and C.A. Dukes, Phys. Rev. Lett. 76, 2547 (1996).

14. Handbook of Auger Electron Spectroscopy - Physical Electronics Inc., Eden Praire, Minnesota, USA, 1976

15. D.M. Roessler, W.C. Walker, Phys. Rev. 159, 733 (1967).

16. V.E. Henrich, G. Dresselhaus, H.J. Zieger Phys. Rev. B 22, 4764 (1980).

17. J.C. Tucek and R.K. Champion, Surf. Sci. 382, 137 (1997) and references therein.

18. H. Hagstrum in Inelastic Ion Surface Collisions, Ed. N.H. Tolk,J.C. Tully, W. Heiland and C. White (Academic Press, New York, 1977) p.1

19. S.J. Yoon, I. Lee, J-W. Lee and B. Oh, Jpn. J. Appl. Phys. 40, 809 (2001)

20. P. Roncin, J. Villette, J.P. Atanas, H. Khemliche, Phys. Rev. Lett. 84, 864 (1999).

21. J.C. Lancaster, F.J. Kontur, G.K. Walters, and F.B. Dunning, Phys. Rev. A 64, 54901 (2001).

22. Y.T. Matulevich, T.J. Vink, and P.A. Zeijlmans van Emmichoven, Phys. Rev. Lett. 89, 167691 (2002)

23. R.A. Baragiola, M. Shi, R.A. Vidal, and C.A. Dukes, Phys. Rev. B 58, 13212 (1998).

24. M. Barat and W. Lichten, Phys. Rev. A6, 211 (1972).

25. R. Souda, et al., Surface Sci. 343, 104 (1995).

26. P. A. Cox and A. A. Williams, Surf. Sci. 175, L782 (1986)

27. C. Satoko, M. Tsukada, and H. Adachi, J. Phys. Soc. Jpn. 45, 1333 (1978)

28. S. Russo and C. Noguera, Surface Sci. 262, 245 (1992)

29. Our diagram differs from that of P. Wurz, J. Sarnthein, W. Husinsky, G. Betz, P. Nordlander, and Y. Wang, Phys. Rev. B 43, 6729 (1998) for LiF, where a surface potential well is proposed by locating the vacuum level several $\mathrm{eV}$ above the conduction band minimum at the surface. Such a diagram is not consistent with the similarity of ionization energy and minimum electron energy loss measurements also observed for $\mathrm{LiF}^{26}$

30. C. Bandis and B.B. Pate, Phys. Rev. Lett. 74, 777 (1995)

31. V.M. Asnin and I.L. Krainsky, Appl. Phys. Lett. 73, 3727 (1998) 